\title{
Design and simulation study of the translational-knee lower extremity exoskeleton
}

\author{
Yang Li, Xiaorong Guan, Yifei Tong, Cheng Xu \\ School of Mechanical Engineering 105, Nanjing University of Science and Technology, Nanjing 210094, China, \\ E-mail: 562339471@qq.com
}

cross $^{\text {ref }}$ http://dx.doi.org/10.5755/j01.mech.21.3.8795

\section{Introduction}

Human lower extremity exoskeleton is a kind of wearable intelligent robot [1] that has capability of carrying a payload and can supplement human intelligence with the strength. It can be used for the soldiers to bear heavy payload when marching [2], so it can reduce the burden of the pilot in real time. Besides, it's capable of transporting heavy materials over rough terrain or up staircases.

At present, without exception, DOF of all lower limb exoskeleton was designed as revolute joints. The earlier lower extremity exoskeleton developed by University of Berkeley called BLEEX. Adam Zoss [3, 4] et al. proposed the biomechanical design for the BLEEX that has 7 revolute DOF per leg, four of which are powered by linear hydraulic actuators. And the medical exoskeleton designed by University of Berkeley only has knee and hip joints that are actuated in the sagittal plane using hydraulic actuators [5, 6]. The lower extremity exoskeleton developed by Tomoyoshi Kawabata et al. of Tsukuba University called HAL [7]. In the earlier stages, HAL has been developed to assist the lower half body; the newly made upper half body of the robot suit is directly attached to it. For each half, left and right, articulation of the lower half body of the robot suit is achieved by three single-axis revolute joints. The lower extremity exoskeleton developed by K. H. Low [8] et al. of Singapore Nanyang Technological University called NTU. The NTU is anthropomorphic and ergonomic, not only in shape but also in function. On the one hand, it should be analogous to the human lower limb in the case of joint positions and distribution of DOF; on the other hand, the actuators in the exoskeleton leg should be allocated in the corresponding position to the representative muscles in human leg, in order to simulate the function of the muscles during the process of human walking.

In addition, the effectiveness inevitably needs to be verified when designing the lower extremity exoskeleton. And at present, the effectiveness is proved through experiments by most researchers. A novel adaptive foot system to enhance the required stability of lower extremity exoskeletons as an add-on device was proposed by Jungwon Yoon et al. [9]. And Experiments have been conducted to prove the effectiveness of the adaptive wearable device for postural and gait stability. An under-actuated wearable exoskeleton system to carry a heavy load was proposed by S. N. Yu [10] et al. And several experiments were performed to evaluate the performance of the proposed exoskeleton system by measuring the electromyography signal of the wearer's muscles while walking on level ground and climbing up stairs with 20 to $40 \mathrm{~kg}$ loads, respectively.

Differently in this paper, the commonly used rotation knee joint for lower extremity exoskeleton was optimized as translational joint. The translational-knee joint will make the structure of lower extremity exoskeleton more compact and simple, as well as it will make the implementation of control strategy of the lower extremity exoskeleton more convenient. After the dynamic mathematical model of the translational-knee lower extremity exoskeleton was analyzed, the three cases for the human body: without bearing load, independently bearing load and wear the translational-knee lower extremity exoskeleton bearing load were simulated and compared. At last, the power support effect of the exoskeleton was analyzed, and then the feasibility of this translational-knee joint was verified.

\section{Design and modelling of translational-knee lower extremity exoskeleton}

The muscles of human can provide large tension to pull bones to move around the joints, just like the lever [11]. However, the bones and muscles of human body have certain defects. Bones have supporting capacity, but can't stretch out and draw back to provide pressure and tension. Muscles can provide tension, but don't have supporting capacity. However, the linear hydraulic actuators or pneumatic actuators can make up the above shortage. So it is not necessary to design humanoid machine completely according to the joint of human body, such as the lower extremity exoskeleton.

After the structure model design of lower extremity exoskeleton is completed, the kinematics and dynamics parameters of the model in the whole dynamic process can be effectively obtained by modeling and simulating of its mathematical model, and then the feasibility and practicability of the design can be proved.

\subsection{Structure design of translational-knee lower extremity exoskeleton}

Because of the particularity that the lower extremity exoskeleton should be worn on the body of human and the necessity of ensuring maximum safety and minimum collisions with the environment and operator, it's essential to consider the anthropomorphic design in the structure design of lower extremity exoskeleton [3]. Anthropomorphic design can simplify a lot of problems, but also can cause mind-set in innovation.

For example, the heel position relative to the hip joint can be adjusted to realize all kinds of gait of human 
body by rotating the hip joint, knee joint and ankle joint. But for the end control of heel [12], the same control function can also be achieved if the knee joint of the lower extremity exoskeleton is designed as a translational joint.

The translational-knee can be realized by using a set of hydraulic cylinder instead of leg. It will make the structure of the lower extremity exoskeleton more compact, as well as it will make the implementation of control strategy of the lower extremity exoskeleton more convenient. The simplified model is shown in Fig. 1.

The coordinate system usually used for the movement of human body is as follows: the $X$ axis points to the forward direction of human body, the $\mathrm{Z}$ axis is vertical upward, and the $\mathrm{Y}$ axis can be determined by the right hand rule. Generally, the $\mathrm{Y}$ axis is referred to as flexion/extension axis; the $\mathrm{X}$ axis is referred to as adduction/abduction axis, and the $\mathrm{Z}$ axis is referred to as rotation axis [3].

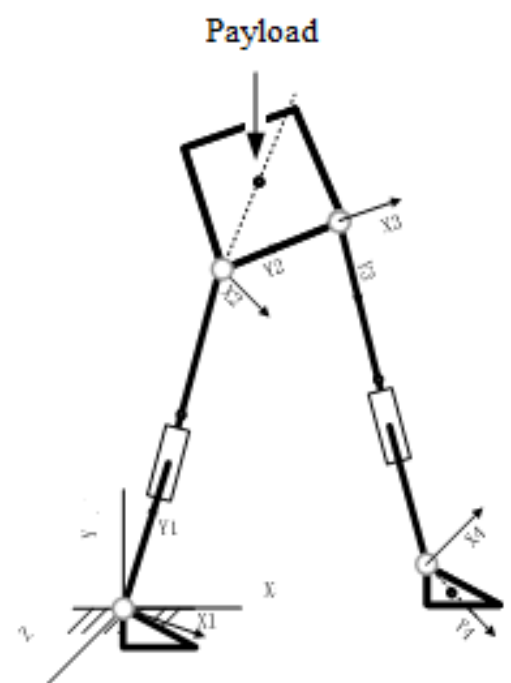

Fig. 1 Translational-knee lower extremity exoskeleton

The DOF and actuated conditions of the translational-knee lower extremity exoskeleton structure model are designed as follows: the hip joint has DOF on flexion/extension axis and adduction/abduction axis, and different from the DOF on flexion/extension axis that is actuated by hydraulic cylinder the DOF on adduction / abducttion axis is in passive state; each leg of the lower extremity exoskeleton is a set of hydraulic cylinder, so the knee joint has a translational DOF; the ankle joint has all the three rotational DOF, and all the three DOF are in passive state.

\subsection{Kinematics and dynamics modelling}

The Lagrange mechanics theory is often used to analyze the dynamic mathematical model of the robot operation, which is also the theoretical basis of dynamic simulation software like Adams.

In the process of walking, the left and right leg is periodically in stance configuration and swing configuration. The kinematics and dynamics analysis is done by taking the phase that one leg is in the stance configuration while the other leg is in swing as an example.

\subsubsection{Definition of the coordinate system}

The system coordinate system is the same as the usually used coordinate system for the movement of hu- man body. The local coordinate system of each joint of the lower extremity exoskeleton is defined as follows: the $\mathrm{Y}$ axis is from each joint point to the next joint; the $\mathrm{X}$ axis is parallel to the XY plane of the system coordinate system; the $\mathrm{Z}$ axis can be determined by the right hand rule.

\subsubsection{Space coordinate transformation matrix}

Assuming there is a coordinate system A and a coordinate system $B$. The coordinates of point $P$ is $P_{A}=\left(\begin{array}{lll}P_{x a} & P_{y a} & P_{z a}\end{array}\right)$ in the coordinate system $\mathrm{A}$; is $P_{B}=\left(\begin{array}{lll}P_{x b} & P_{y b} & P_{z b}\end{array}\right)$ in the coordinate system B. And the coordinates of the origin of coordinate system $B$ is $\left(O_{B}\right)_{A}=\left(\begin{array}{lll}a & b & c\end{array}\right)$ in the coordinate system A.

$\cos \left(x_{a} x_{b}\right)$ is the angle cosine of the $\mathrm{X}$ axis of coordinate system $\mathrm{A}$ with the $\mathrm{X}$ axis of coordinate system $\mathrm{B}$.

So:

$$
\begin{aligned}
& {\left[\begin{array}{l}
P_{x a} \\
P_{y a} \\
P_{z a} \\
1
\end{array}\right]=\left[\begin{array}{ccccc}
\cos \left(x_{b} x_{a}\right) & \cos \left(y_{b} x_{a}\right) & \cos \left(z_{b} x_{a}\right) & a \\
\cos \left(x_{b} y_{a}\right) & \cos \left(y_{b} y_{a}\right) & \cos \left(z_{b} y_{a}\right) & b \\
\cos \left(x_{b} z_{a}\right) & \cos \left(y_{b} z_{a}\right) & \cos \left(z_{b} z_{a}\right) & c \\
0 & 0 & 0 & 1
\end{array}\right]\left[\begin{array}{l}
P_{x b} \\
P_{y b} \\
P_{z b} \\
1
\end{array}\right]} \\
& \text { Simply: }\left[\begin{array}{l}
P_{a} \\
1
\end{array}\right]=\left[T_{a b}\right]\left[\begin{array}{l}
P_{b} \\
1
\end{array}\right]=\left[\begin{array}{ll}
R_{a b} & S_{a b} \\
0 & 1
\end{array}\right]\left[\begin{array}{l}
P_{b} \\
1
\end{array}\right]
\end{aligned}
$$

Therefore the space coordinate transformation matrix of every joint coordinate system relative to the system coordinate system can be gotten by the above formula.

\subsubsection{Velocity and angular velocity of the center of mass}

Assuming that there is a point $P$ in the " $i$ ” joint coordinate system and its coordinate vector is $\left[P_{i i}\right]$ in the " $i$ " joint coordinate system; is $\left[P_{i 0}\right]$ in the system coordinate system.

$$
\text { So: }\left[P_{i 0}\right]=\left[S_{i 0}\right]+\left[R_{i 0}\right]\left[P_{i i}\right] \text {. }
$$

Take a derivative with respect to time:

$$
\left[\dot{P}_{i 0}\right]=\left[\dot{S}_{i 0}\right]+\left[R_{i 0}\right]\left[\dot{P}_{i i}\right]+\left[\dot{R}_{i 0}\right]\left[P_{i i}\right] \text {. }
$$

and $\left[\dot{R}_{i 0}\right]=\left[d R_{i 0}\right] / d t=\left[\Delta_{i 0}\right]\left[R_{i 0}\right] / d t,[\Delta]$ is called "differential operator":

$$
\left[\Delta_{i 0}\right]=\left[\begin{array}{ccc}
0 & -\delta_{i 0 z} & \delta_{i 0 y} \\
\delta_{i 0 z} & 0 & -\delta_{i 0 x} \\
-\delta_{i 0 y} & \delta_{i 0 x} & 0
\end{array}\right] . \delta_{i 0 x}, \delta_{i 0 y}, \delta_{i 0 z}
$$

respectively express the differential rotation angle of each coordinate axis of the joint coordinate system relative to the system coordinate system.

So:

$\left[\omega_{i 0}\right]=\left[\Delta_{i 0}\right] / d t=\left[\begin{array}{ccc}0 & -\omega_{i 0 z} & \omega_{i 0 y} \\ \omega_{i 0 z} & 0 & -\omega_{i 0 x} \\ -\omega_{i 0 y} & \omega_{i 0 x} & 0\end{array}\right]=\left[\begin{array}{ccc}0 & -\omega_{i 0 z} & 0 \\ \omega_{i 0 z} & 0 & -\omega_{i 0 x} \\ 0 & \omega_{i 0 x} & 0\end{array}\right]$

and it’s called “angular velocity operator”: 


$$
\left[\dot{R}_{i 0}\right]=\left[d R_{i 0}\right] / d t=[\Delta]\left[R_{i 0}\right] / d t=\left[\omega_{i 0}\right]\left[R_{i 0}\right]
$$

Then:

$$
\begin{gathered}
{\left[v_{i 0}\right]=\left[\dot{P}_{i 0}\right]=\left[\dot{S}_{i 0}\right]+\left[R_{i 0}\right]\left[\dot{P}_{i i}\right]+\left[\omega_{i 0}\right]\left[R_{i 0}\right]\left[P_{i i}\right] .} \\
\text { Besides: }\left[\omega_{i i}\right]=\left[\begin{array}{lll}
\omega_{i 0 x} & \omega_{i 0 y} & \omega_{i 0 z}
\end{array}\right]^{T} .
\end{gathered}
$$

\subsubsection{Gravitational potential energy and kinetic energy}

Assuming $G$ represents the center of gravity of each part of the lower extremity exoskeleton, so the gravitational potential energy of each part of the lower extremity exoskeleton is $N_{i}=m_{i} g y_{G i 0}$. Therefore the total potential energy $N$ of the system can be obtained on the basis of above.

Besides, the translational kinetic energy of each part is $K_{G i 0}^{v}=\frac{1}{2} m_{G i}\left[v_{i 0}\right]^{\mathrm{T}}\left[v_{i 0}\right]$; the rotational kinetic energy of each part is $K_{G i i}^{\omega}=\frac{1}{2} I^{i i}\left[\begin{array}{lll}\omega_{i 0 x}{ }^{2} & \omega_{i 0 y}{ }^{2} & \omega_{i 0 z}{ }^{2}\end{array}\right]^{\mathrm{T}}$. So the total kinetic energy can be obtained by $K=K^{v}+K^{\omega}$.

\subsubsection{The Lagrange equation}

The Lagrange function is $L=K-N$.

According to the Lagrange equation, the torques of rotational joints and the forces of translational joints of the lower extremity exoskeleton in the whole process of dynamic operation can be obtained.

$$
\left\{\begin{array}{l}
T_{\alpha_{i}}=\frac{d}{d t} \frac{\partial L}{\partial \dot{\alpha}_{i}}-\frac{\partial L}{\partial \alpha_{i}} \ldots \ldots \ldots \ldots . . .(\text { Rotational joint }) \\
F_{l_{i}}=\frac{d}{d t} \frac{\partial L}{\partial \dot{l}_{i}}-\frac{\partial L}{\partial l_{i}} \ldots \ldots \ldots \ldots \ldots \text { (Translational joint) }
\end{array}\right.
$$

\subsection{Simulation process}

When carrying out the Adams simulation, the velocity and acceleration of each joint of the lower extremity exoskeleton can be calculated based on the kinematics theory. And then, the energy and force of each joint can be work out based on the dynamics theory. So the power support effect of the lower extremity exoskeleton to human body when bearing heavy payload can be effectively work out by simulating the models.

The following three cases were combined to compare for the power support effect: $\mathrm{O}, 1$ the man-machine coupled model of human body, lower extremity exoskeleton and payload; O, 2 the model of human body directly bearing payload; O, 3 the model of human body without bearing payload. The flow chart of simulation is as shown in Fig. 2.

When simulating: all DOF on human body joints are actuated by the data curve (the curve of angle changing with time) that are obtained in the form of independently collected CGA (Clinical Gait Analysis) data [13]; only the four set of hydraulic cylinders of the lower extremity exoskeleton are actuated by pressure curve; all other DOF are in passive state. In addition, the pilot and the lower extremity exoskeleton are set rigid mechanical connections

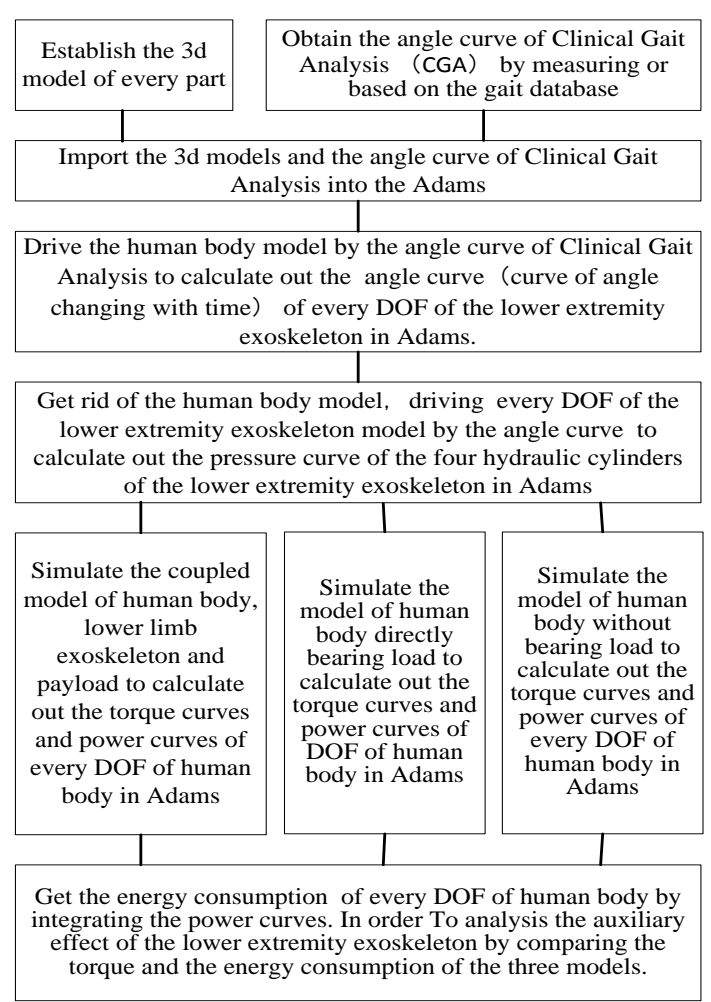

Fig. 2 Flow chart of simulation

at the torso and feet. So the payload and the dead-weight of exoskeleton can be partly transferred to the human body as interaction forces through the connections.

\section{Simulation of the man-machine coupled model}

\subsection{Establish the man-machine coupled model}

In Sport Biomechanics, human body model can be simplified as multi-rigid-body system consisting of multiple rigid links [14]. The man-machine coupled model is shown in Fig. 3.

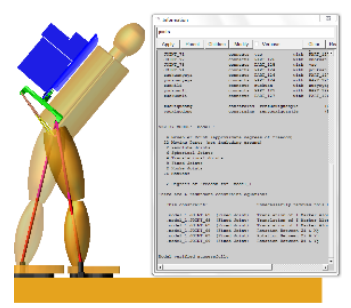

Fig. 3 Man-machine coupled model

The above simulation model includes: 22 parts, 4 translational joints, 5 spherical joints, 2 hooke joints, 2 revolute joints, 9 fixed joints, 2 rotational motions, 4 general motions, 4 force motions, and 14 splines.

The whole process of the man-machine coupled model that moves from the state shown in figure 3 to one step forward was simulated. Parameters are as follows:

- payload weigh: $100 \mathrm{~kg}$;

- time of the process: $0.25 \mathrm{~s}$;

- half stride: $377.75 \mathrm{~mm}$;

- walking speed: $1.511 \mathrm{~m} / \mathrm{s}$.

The simulated whole moving process is as shown in Fig. 4. 


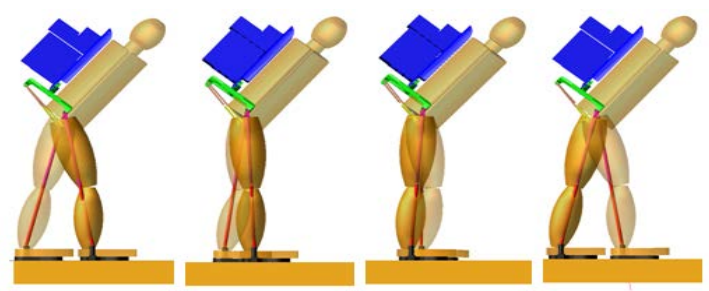

Fig. 4 Simulated whole moving process

\subsection{Determinate the drive curve}

Every DOF of human body model were driven by the CGA angle curve, and all DOF of the lower extremity exoskeleton are in passive state, so the angle curve (curve of angle changing with time) of every DOF of the lower extremity exoskeleton can be calculated in Adams.

Then get rid of the human body model, and just using the angle curve of every DOF to drive the lower extremity exoskeleton model, then the pressure curve (as shown in Fig. 5) for the four hydraulic cylinders of the lower extremity exoskeleton can be calculated out in Adams.

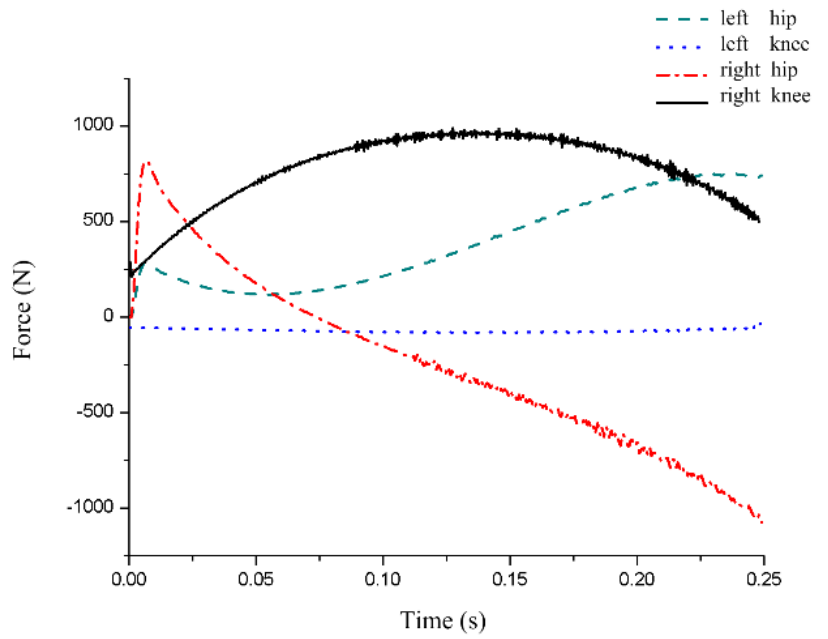

Fig. 5 Pressure curve of the four hydraulic cylinders

In the whole process, the right leg is in stance configuration and the left leg is in swing configuration. It can be seen from the Fig. 5 that the biggest pressure of the hydraulic cylinders is approximately equal to the sum of the weight of payload $(100 \mathrm{~kg})$ and the lower extremity exoskeleton $(10 \mathrm{~kg})$. It can also be seen that the pressure of the hydraulic cylinders of right leg is larger than that of left leg, because the whole payload forces are transferred to the ground by the stance leg. Besides, the needed pressure of the hydraulic cylinders of left hip joint is much larger than that of left knee joint, because the left hip needs to swing the whole leg other than the left knee just needs to stretch out or draw back the shank.

\subsection{Driving torque of human joints}

The kinematic and dynamic parameters (e.g. angle, angular velocity, angular acceleration, torque, power) of human body can be obtained by doing the multi-body dynamics simulation of the man-machine coupled model using Adams. The driving torques of human joints of the whole process is shown in Figs. 6 and 7.

It can be seen from Figs. 6 and 7 that the driving

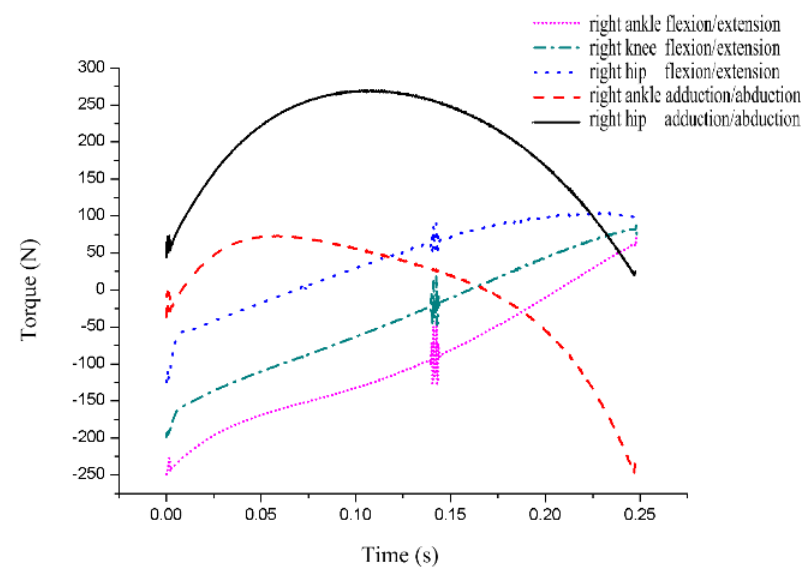

Fig. 6 Driving torques of right joints

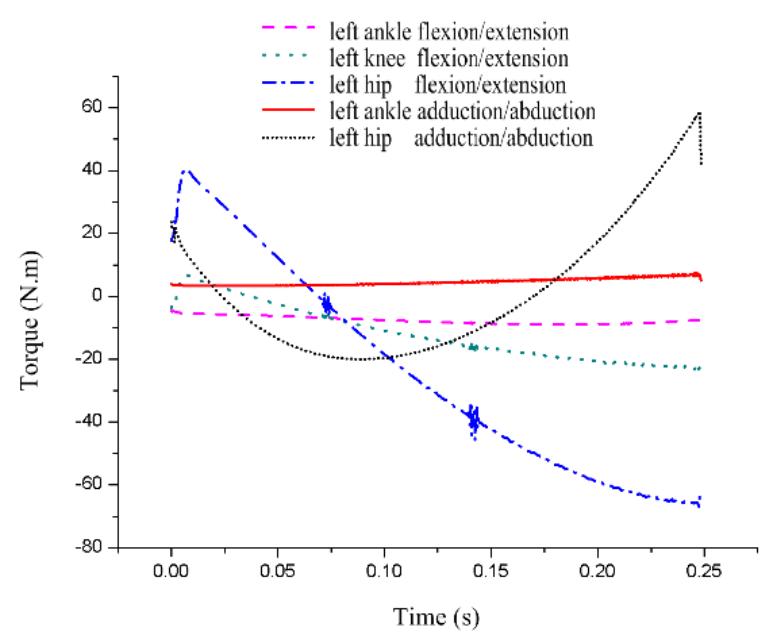

Fig. 7 Driving torques of left joints

torques of right leg joints of human body are bigger than that of left leg joints, because the right leg needs to support the whole payload forces. Besides, the driving torque of each DOF of right leg joint is similar other than the driving torque of left hip joint are larger than other left joints.

\subsection{Driving power of human joint}

The driving powers of every human joint during the whole process are shown in Figs. 8 and 9.

Similar to the driving torque, it can be seen that the driving powers of right leg joints of human body is bigger than that of left leg joints. But the driving powers of hip joint are bigger than other joints in both the two human legs.

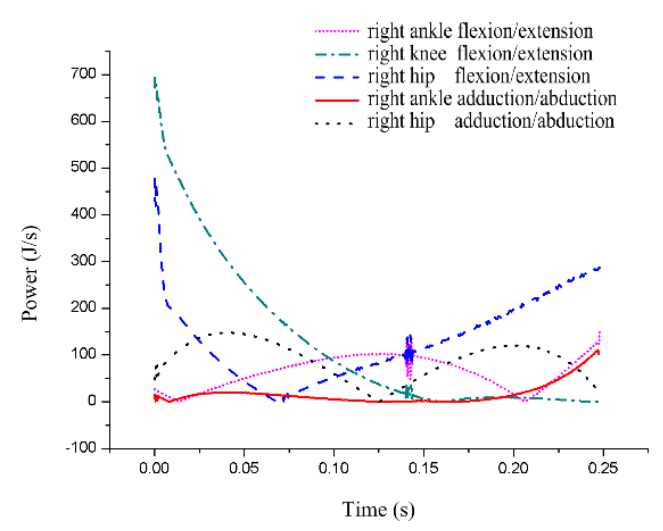

Fig. 8 Driving powers of right joints 


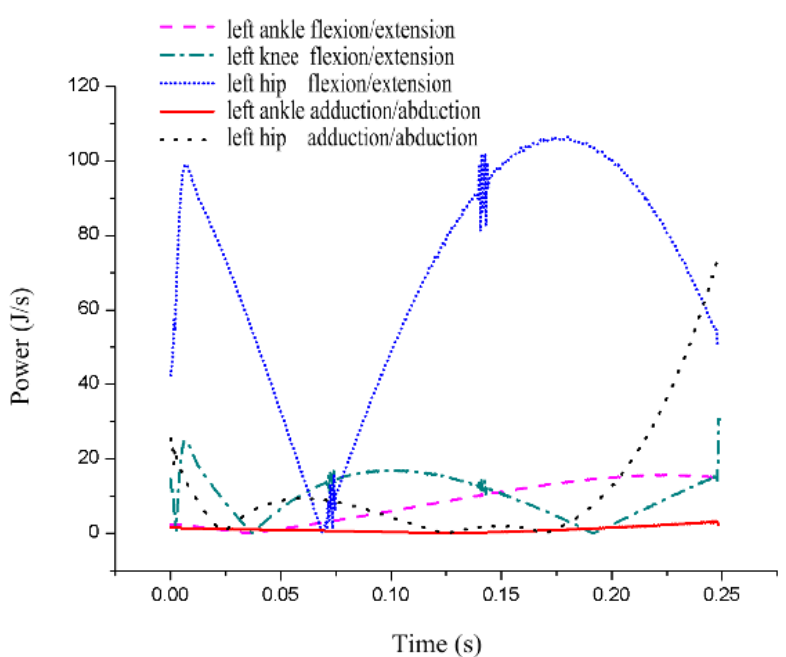

Fig. 9 Driving powers of left joints

\section{Compare of different cases simulation}

Different cases were combined to compare for the power support effect of the lower extremity exoskeleton, the model of human body directly bearing payload is shown in Fig. 10, a and the model of human body without bearing payload is shown in Fig. 10, b.

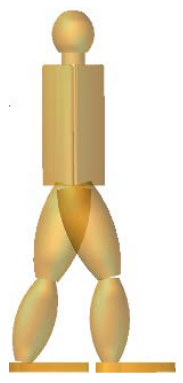

a

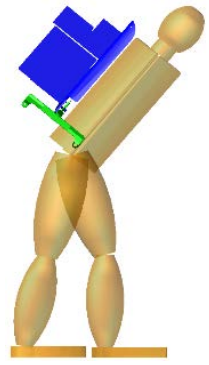

b
Fig. 10 Human body: a - without bearing payload; b - directly bearing payload

\subsection{Compare of driving torque}

The driving torques of human joints of different cases is shown in Table 1.

Table 1

Driving torque of each joint of different models

\begin{tabular}{|l|cc|cc|cc|}
\hline \multicolumn{1}{|c|}{ Case } & \multicolumn{2}{|c|}{ I } & \multicolumn{2}{c|}{ II } & \multicolumn{2}{c|}{ III } \\
\hline \multicolumn{1}{|c|}{ DOF } & fle/ext & add/abd & fle/ext & add/abd & fle/ext & add/abd \\
\hline Torque of right ankle, Nm & $-58 \sim-33$ & $-84 \sim 26$ & $-249 \sim 60$ & $-249 \sim 72$ & $-249 \sim 89$ & $-264 \sim 36$ \\
Torque of right knee, Nm & $-55 \sim 26$ & & $-198 \sim 82$ & & $-208 \sim 139$ & \\
Torque of right hip, Nm & $-44 \sim 109$ & $-3 \sim 122$ & $-124 \sim 99$ & $20 \sim 269$ & $-118 \sim 177$ & $0.2 \sim 217$ \\
\hline Torque of left ankle, Nm & $-9 \sim-5$ & $0 \sim 4$ & $-9 \sim-5$ & $3 \sim 7$ & $-9 \sim 5$ & $0 \sim 4$ \\
Torque of left knee, Nm & $-37.9 \sim-3$ & & $-17 \sim 7$ & & $-37.9 \sim-3$ & \\
Torque of left hip, Nm & $-107 \sim 21$ & $-20 \sim 58$ & $-66 \sim 41$ & $-20 \sim 58$ & $-107 \sim 17$ & $-20 \sim 58$ \\
\hline
\end{tabular}

Notes: fle/ext means flexion/extension; add/abd means adduction/abduction; I: Human body; II: Human body+ lower extremity exoskeleton+ payload; III: Human body + payload

As for the swing leg (left leg), it can be seen from Table 1 that the range of driving torque of each joint of different cases is similar. As for the stance leg (right leg), the range of each driving torque of flexion/extension joints of case II is smaller than case III, but the range of each driving torque of adduction/abduction joints of case II is even slightly larger than case III. So the power support effect of lower extremity exoskeleton cannot be clearly reflected by the range of driving torque.

\subsection{Compare of energy consumption}

The energy consumption in the whole process (as shown in Table 2) of each DOF of human body can be obtained by integrating the power curves.

It can be found by statistics that the power consumption of $63 \mathrm{~kg}$ male is about $3 \mathrm{kcal} / \mathrm{min}$ when walking at $1.33 \mathrm{~m} / \mathrm{s}$ speed, $6 \mathrm{Kcal} / \mathrm{min}$ at $1.77 \mathrm{~m} / \mathrm{s}$ speed and $10 \mathrm{Kcal} / \mathrm{min}$ at $2.7 \mathrm{~m} / \mathrm{s}$ speed. When walking at $1.51 \mathrm{~m} / \mathrm{s}$, a normal walking speed, fewer muscles are mobilized, and energy is mainly consumed to adjust the gravity center and focuses on the legs [15]. The established human body model weighs about $70 \mathrm{~kg}$ without arms. It can be calcula-

Energy consumption of each joint of different models

\begin{tabular}{|c|c|c|c|c|c|c|}
\hline Case & \multicolumn{2}{|l|}{$\mathrm{I}$} & \multicolumn{2}{|l|}{ II } & \multicolumn{2}{|l|}{ III } \\
\hline DOF & fle/ext & add/abd & fle/ext & add/abd & fle/ext & add/abd \\
\hline Energy of right ankle, $\mathrm{J}$ & 11.9 & 1.7 & 15.6 & 4.0 & 18.1 & 4.4 \\
\hline Energy of right knee, $\mathrm{J}$ & 11.8 & & 31.2 & & 34.7 & \\
\hline Energy of right hip, J & 28.3 & 10.0 & 32.3 & 22.3 & 50.5 & 36.5 \\
\hline Total energy of right leg, J & 63.7 & & 105.4 & & 144.2 & \\
\hline Energy of left ankle, $\mathrm{J}$ & 2.0 & 0.1 & 2.1 & 0.3 & 2.0 & 0.1 \\
\hline Energy of left knee, J & 3.6 & & 2.6 & & 3.6 & \\
\hline Energy of left hip, J & 25.0 & 3.0 & 17.4 & 2.9 & 25.0 & 3.0 \\
\hline Total energy of left leg, $\mathrm{J}$ & 33.7 & & 25.3 & & 33.7 & \\
\hline Total energy, J & 97.4 & & 130.7 & & 177.9 & \\
\hline
\end{tabular}


ted out from Table 2 that the total energy consumption of the human legs in the walking process is $(63.7+33.7) \mathrm{J} / 0.25 \mathrm{~s}=5.7 \mathrm{Kcal} / \mathrm{min}$, which is coincident with the statistics.

It can be seen that the energy consumption of right leg of case II is much lower than that of case III, and the energy consumption of left leg of case II is even slightly lower than that of model I. The total energy consumption of model III is 177.9-97.4 $=80.5 \mathrm{~J}$ more than model I and the model II is $130.7-97.4=33.3 \mathrm{~J}$ more than model I. So the lower extremity exoskeleton can reduce the total energy consumption of human body by (80.533.3) $/ 80.5=58.6 \%$ for extra bearing $100 \mathrm{Kg}$ payload.

From what has been discussed above, the designed translational-knee lower extremity exoskeleton can reduce the total energy consumption of human body in the process of walking. Therefore the translational-knee lower extremity exoskeleton can provides the ability to carry significant payloads with minimal effort to its operator.

\section{Conclusion}

To seek more compact structure and power support effect theoretical basis for lower extremity exoskeleton:

1. The structure of lower extremity exoskeleton was analyzed, and the translational-knee lower extremity exoskeleton was designed. The translational-knee will make the structure of the lower extremity exoskeleton more compact, as well as it will make the implementation of control strategy of the lower extremity exoskeleton more convenient

2. The kinematics and dynamics mathematical model of the designed translational-knee lower extremity exoskeleton was analyzed. And the Lagrange mechanics theory can provide theoretical basis of dynamic simulation software like Adams

3. The 3D model of every part of the translational-knee lower extremity exoskeleton was established. And the $3 \mathrm{~d}$ model is a requirement of the dynamic simulation.

4. The dynamic simulation by using Adams was carried out, and the power support effects of different cases were compared and analyzed. It can be seen that the energy consumption can obviously reflect the power support effect of the translational-knee lower extremity exoskeleton, because the lower extremity exoskeleton can reduce the total energy consumption of human body by $58.6 \%$ for extra bearing $100 \mathrm{Kg}$ payload in the process of walking

Future work: firstly, a feasible control strategy that is appropriate for the translational-knee lower extremity exoskeleton should be analyzed and proposed. Then, the joint simulation by using Adams and Matlab/Simulink for the control strategy will be carried out, so the feasibility of the control strategy will be proved. At last, a physical prototype will be developed, and then a variety of related experiments will be performed based on the physical prototype.

\section{References}

1. Zhang Jiafan, Chen Ying, Yang Canjun. 2011. Manmachine Intelligent Systems of Flexible Exoskeletons. Science press: 5-6.

2. Yang Zhiyong, Gu Wenjing, Zhang Jing, Gui Lihua.
2013. Force Control Theory and Method of Soldier Load Carrying Exoskeleton Suit. National defense industry press: 1-5.

3. Adam Zoss, H. Kazerooni, Andrew Chu. 2005. On the mechanical design of the Berkeley lower extremity exoskeleton (BLEEX), IEEE Intelligent Robots and Systems, Edmonton, Canada: 3132-3139.

4. Adam Zoss, H.Kazerooni, Andrew Chu. 2006. Biomechanical design of the Berkeley lower extremity exoskeleton (BLEEX), IEEE/ASME Transactions on Mechatronics 11(2): 128-138.

http://dx.doi.org/10.1109/TMECH.2006.871087.

5. Tim A. Swift, Katherine A. Strausser, Adam Zoss, H. Kazerooni. 2010. Control and experimental results for post stroke gait rehabilitation with a prototype mobile medical exoskeleton, Proceedings of the ASME 2010 Dynamic Systems and Control Conference, Cambridge, Massachusetts, USA: 12-15.

6. Katherine A. Strausser, H. Kazerooni. 2011. The development and testing of a human machine interface for a mobile medical exoskeleton, IEEE/RSJ International Conference on Intelligent Robots and Systems, San Francisco, USA: 25-30.

7. Hozumi Satoh, Tomoyoshi Kawabata, Yoshiyuki Sankai. 2009. Bathing care assistance with robot suit HAL. IEEE International Conference on Robotics and Biomimetics, Guilin, China: 19-23.

http://dx.doi.org/10.1109/robio.2009.5420697.

8. K. H. Low, Xiaopeng Liu, Haoyong Yu. 2005. Development of NTU wearable exoskeleton system for assistive technologies, IEEE International Conference on Mechatronics \& Automation Niagara Falls, Canada: 1099-1106.

9. Jungwon Yoon, R. Prasanth Kumar, Abdullah Ozer. 2011. An adaptive foot device for increased gait and postural stability in lower limb orthoses and exoskeletons, International Journal of Control, Automation, and Systems 9(3): 515-524. http://dx.doi.org/10.1007/s12555-011-0311-3.

10. Yu, S.N.; Lee, H.D.; Lee, S.H.; Kim, W.S.; Han, J.S.; Han C.S. 2012. Design of an under-actuated exoskeleton system for walking assist while load carrying, Advanced Robotics 26(5/6): 561-580. http://dx.doi.org/10.1163/156855311X617506.

11. Huang Xiaolin. 2013. Kinesiology. People's medical press: 22-23.

12. Kazerooni, H.; Jean-Louis Racine, Lihua Huang. 2005. On the control of the Berkeley lower extremity exoskeleton (BLEEX), IEEE International Conference on Robotics and Automation, Barcelona: 4364-4371. http://dx.doi.org/10.1109/robot.2005.1570790.

13. Center for Biometrics and Security Research. CASIA Gait Database. http://www.cbsr.ia.ac.cn/china /Gait\%20 Databases\%20CH.asp

14. The national sports colleges and universities teaching committee. 2014, Sports Biomechanics. People's sport press: $1-2$.

15. Dai Jiansong, Li Jing, Gu Zhongke Sun Biao. 2006. The calculation of energy consumption of walking and daily physical activity, Journal of Sport Science 26(11): 91-94. 
Li Yang, Guan Xiaorong, Tong Yifei, Xu Cheng

DESIGN AND SIMULATION STUDY OF THE TRANSLATIONAL-KNEE LOWER EXTREMITY EXOSKELETON

S u m m a r y

In order to make the structure of lower extremity exoskeleton more compact and simple, as well as to provide theoretical basis for the power support effect of lower extremity exoskeleton, the translational-knee lower extremity exoskeleton was designed and the simulation by using Adams was carried out. By comparing the simulation results of three different cases, it can be concluded that the torque range of each degree of freedom (DOF) of the human body does not shrink much by wearing the lower extremity exoskeleton; but the energy consumption in the process can be reduced by $58.6 \%$ for extra bearing $100 \mathrm{Kg}$ payload. It proves that the translational-knee lower extremity exoskeleton can effectively help human body bear heavy payload, and can reduce the energy consumption of human body in the process of walking.

Keywords: lower extremity exoskeleton; translational knee; dynamics simulation; bearing payload; power support effect.

Received November 29, 2014 Accepted March 15, 2015 